\title{
Gene Feature
}

National Cancer Institute

\section{Source}

National Cancer Institute. Gene Feature. NCI Thesaurus. Code C13445.

The structural elements that comprise a gene or transcription unit. Gene structures include the transcription initiation site, the protein coding sequences, intervening sequences, and the transcription termination or polyadenylation sites as well as the regulatory elements (enhancers, silencers, and the promoter) that lie outside the boundaries of the transcribed unit. 